\title{
Adaptive Tracking Control for a Class of Nonlinear Systems with Quantized Input
}

\author{
Xiaowei Yu, a and Yan $\operatorname{Lin}^{2}$ \\ ${ }^{1,2}$ School of Automation, Beijing University of Aeronautics and \\ Astronautics, Beijing, China \\ axiaoweiyueel@126.com
}

Keywords: Nonlinear systems, Quantization, Nussbaum-gain, Adaptive control.

Abstract. We design an adaptive quantized tracking control for a class of nonlinear systems with unknown parameter combined with quantized input. It is proved that by using the proposed method, all signals of the closed-loop system are bounded. Moreover, a very coarse quantization input can be obtained and the tracking error can be made small by choosing suitable design parameters.

\section{Introduction}

Quantized control is motivated by the convergence of controls and communications to address modern engineering applications involving the use of information technology. An important aspect there is to use quantization schemes that have sufficient precision and, at the same time, require low communication rate. Consequently, the study of quantized control came to the attention of researchers, and various results have been obtained for quantized control of linear systems [1-7]. It was proved in [1] that the coarsest quantizer that quadratically stabilizes a single input linear discrete time invariant system is logarithmic. With the sector bound approach, the coarsest quantization densities for stabilization of multivariable systems in both state feedback and output feedback cases were derived in [2]. In [3], with the developed ISS (input-to-state stability) cyclic-small-gain theorem as a tool, the feedback control problem of nonlinear systems in strict-feedback form with state quantizers was studied. Based on the idea of scaling quantization levels, global asymptotic stability was guaranteed in [4] for linear systems with dynamic quantization. In [5], for linear uncertain discrete-time systems with input quantizer, a direct adaptive control framework was developed. More details about quantized control of linear systems can be seen [6].

Compared with the above mentioned achievements, few results about quantized control of nonlinear systems have yet been reported. Systems in practice are always characterized by uncertain and nonlinear in practice. For this reason, much attention has been devoted to adaptive quantized control of uncertain nonlinear systems [7-8]. In [7], for nonlinear uncertain systems with input quantizer, a direct adaptive control framework was developed. However, as pointed out by [8], the stability condition in [7] depends on the control signal and therefore seems hard to be checked in advance. In [8], an adaptive backstepping feedback stabilization scheme was proposed for a class of nonlinear systems. However, to obtain the stability condition, the nonlinear functions must be known and satisfy the global Lipschitz continuity condition with known Lipschitz constants. Nevertheless, for all these schemes, the situation that the quantized input combined with unknown constant was never considered.

The objective of this paper is to design an adaptive quantized control for system (1) such that all the closed-loop signals are bounded even with unknown parameter appeared with the quantized input, and the plant state $x$ tracks a given reference signal $x_{r}=\left[y_{r}, \mathscr{\&}_{r}, \mathrm{~K}, y_{r}^{(n-1)}\right]^{T}$ as closely as possible, where $y_{r}$ and its first $n$ derivatives are known and bounded. The main contributions of this paper are summarized below.

The nonlinear system we considered in this paper have an unknown constant combined with the quantized input, while the coefficients of quantized input of systems in early works are always 1. 
The way to improve the tracking error is clearly illustrated. That is, by properly choosing some design parameters, the tracking error of the quantized control system can be steered to within a small neighborhood of the origin while keeping all the other closed-loop signals bounded.

The remainder of this paper is organized as follows. Section 2 begins with the hysteresis quantizer and the state feedback designed, the main results are given in Section 3, followed by the stability analysis in Section 4. Finally, we conclude in Section 5.

\section{Problem Formulation}

In this paper, we consider the following class of nonlinear systems:

$$
\begin{aligned}
& \underset{\mathbf{\alpha}}{\mathbf{\alpha}}=x_{i+1}, i=1,2, \mathrm{~K}, n-1 \\
& \underset{n}{\boldsymbol{\alpha}}=f(x)+b q(u)+\Delta(t), \\
& y=x_{1},
\end{aligned}
$$

where $y$ is the output and $x$ is the state vector; $b$ is a unknown constant and $\Delta(t)$ is unknown nonlinear function. The input $q(u)$ represents the quantizer and takes the quantized values, where $u$ is the control input signal to be quantized at the encoder side. The hysteresis quantizer is described as follows:

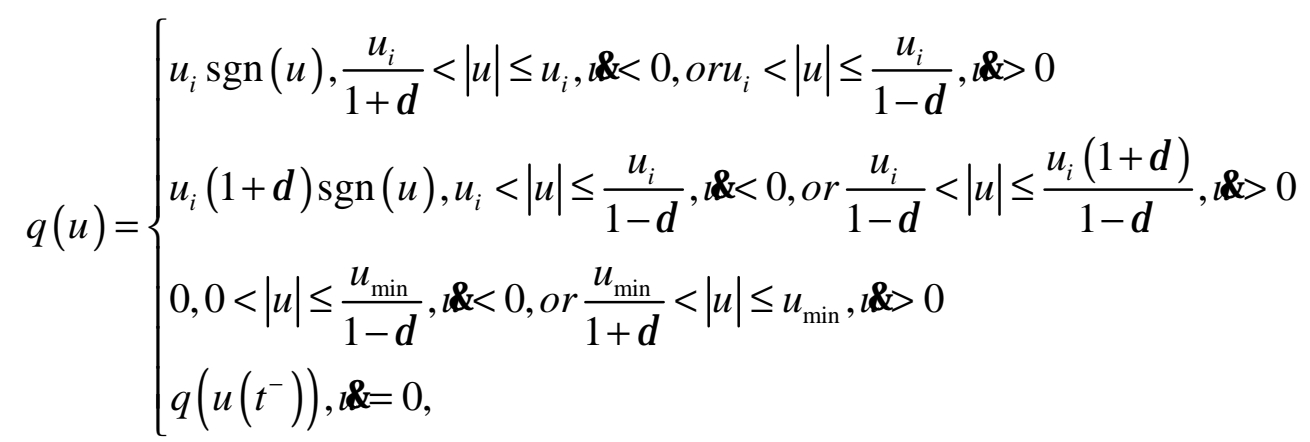

where $u_{i}=\rho^{1-i} u_{\min }$ with integer $i=1,2, \mathrm{~K}$ and parameters $u_{\min }>0$ and $0<\rho<1, \delta=\frac{1-\rho}{1+\rho} . u_{\min }$ determines the size of the dead-zone for $q(u)$.

For the system to be controlled, we make the following assumptions.

A.1: The sign of $b$ is known.

A.2: There exit an unknown constant $\theta$ and a known nonnegative smooth function $g(x)$ such that

$$
|\Delta(x, t)| \leq \theta g(x),
$$

With the above assumptions, the objective is to design an adaptive controller such that the plant state $x$ tracks a given reference signal $x_{r}=\left[y_{r}, \bigotimes_{r}, \mathrm{~K}, y_{r}^{(n-1)}\right]^{T}$ as closely as possible, while all the closed-loop signals remain bounded, where $y_{r}$ and its first $n$ derivatives are known and bounded.

To begin with, we present some preliminary results.

The hysteretic quantizer $q(u)$ is decomposed into a linear part and a nonlinear part as follows:

$$
q(u)=u+d
$$

for which, the following lemma holds.

Lemma 1: The nonlinearity $d(t)$ satisfies the following inequalities.

$$
\begin{aligned}
& d^{2}(t) \leq \delta^{2} u^{2}, \forall|u| \geq u_{\text {min }}, \\
& d^{2}(t) \leq u_{\text {min }}^{2}, \forall|u| \leq u_{\text {min }} .
\end{aligned}
$$

To deal with the unknown parameter, the Nussbaum gain approach is employed in this paper. 
Lemma 2: Let $V(\square)$ and $\zeta(\square)$ be smooth functions defined on $\left[0, t_{f}\right)$ with $V(t) \geq 0, \forall t \in\left[0, t_{f}\right)$ and $N(\square)$ be an even Nussbaum-type function. For $t \in\left[0, t_{f}\right)$, if the following inequality holds:

$$
\left.V(t) \leq c_{0}+\int_{0}^{s} e^{-c_{1}(t-\tau)}[g(\tau) N(\zeta(\tau))+1] \zeta \phi_{(\tau)}\right) d \tau,
$$

Where constant $c_{1}>0, g(t)$ is a time-varying parameter which takes values in the unknown closed intervals $I=\left[l^{-}, l^{+}\right]$with $0 \notin I$, and $c_{0}$ represents some suitable constant, then $V(t), \zeta(t)$ and $\int_{0}^{s} g(\tau) N(\zeta) \zeta \mathcal{\delta}(\tau) d \tau$ must be bounded on $\left[0, t_{f}\right)$.

\section{Control Scheme}

In this section, we will design an adaptive control scheme for the nonlinear system with quantized input.

Let the error be defined as

$$
\begin{gathered}
x_{r}=\left[y_{r}, \bigotimes_{r}, \mathrm{~K}, y_{r}^{(n-1)}\right]^{T}, \\
\beta=x-x_{r},
\end{gathered}
$$

and a filtered tracking error as

$$
e=\left[\lambda_{1}, \lambda_{2}, \mathrm{~K}, \lambda_{n-1}, 1\right] \beta,
$$

where $\lambda_{1}, \lambda_{2}, \mathrm{~K}, \lambda_{n-1}$ are design parameters that make $s^{n-1}+\lambda_{n-1} s^{n-2}+\mathrm{L}+\lambda_{1}$ Hurwitz. Besides, we shall employ positive scalars $\gamma, \sigma$ and $k_{d}$ as design parameters in the subsequent design without restating.

The derivative of $e$, by considering (8) and (9), can be expressed as

$$
\Leftrightarrow f(x)+b q(u)+\Delta-y_{r}^{(n)}+\left[0, \lambda_{1}, \mathrm{~K}, \lambda_{n-1}\right] \beta,
$$

Define the quadratic function

$$
V_{e}=\frac{1}{2} e^{2}
$$

Differentiating $V_{e}$ it can be checked that

$$
\hat{\Sigma}_{e} \leq e f(x)+e b q(u)+|e| \theta g+e Y_{d},
$$

where $Y_{d}=-y_{r}^{(n)}+\left[0, \lambda_{1}, \mathrm{~K}, \lambda_{n-1}\right]$.

Let $\hat{\theta}$ be the estimate of $\theta$, and

Consider the following Lyapunov function

$$
\hat{\theta} \% \theta-\hat{\theta} .
$$

$$
V=V_{e}+\frac{1}{2 \gamma} \theta^{\%},
$$

Differentiating $V$ yields

$$
\varepsilon_{\leq} e f(x)+e b q(u)+|e| \theta g+e Y_{d}-\frac{1}{\gamma} \dot{\theta} \hat{\Theta} .
$$

Finally, the actual control $u$ is chosen as

$$
u=-\frac{\operatorname{sgn}(b) e v^{2}}{(1-\delta) \sqrt{e^{2} v^{2}+\eta^{2}}},
$$

where $\eta$ is a positive design parameter and 


$$
\begin{aligned}
& v=-N(\zeta)\left[c e+f(x)+\hat{\theta} g \tanh \left(\frac{e g}{\varepsilon}\right)+Y_{d}\right], \\
& \zeta \&=c e^{2}+e f(x)+e \hat{\theta} g \tanh \left(\frac{e g}{\varepsilon}\right)+e Y_{d},
\end{aligned}
$$

and the adaptive law $\theta^{\S}$ is chosen as

$$
\theta^{\&}=\gamma e g \tanh \left(\frac{e g}{\varepsilon}\right)-\gamma \sigma \hat{\theta} .
$$

Then, differentiating $V$ yields

$$
\delta \leq e f(x)+e b(u+d)+|e| \theta g+e Y_{d}-\frac{1}{\gamma} \theta^{\circ} \delta .
$$

\section{Main Results}

To have the stability analysis of the system, the following lemma is necessary.

Lemma 3: For any constant $\lambda>0$ and any variable $z$, the following relationship hold

$$
-\frac{z^{2}}{\sqrt{z^{2}+\lambda^{2}}} \leq \lambda-z
$$

We are now in a position to present our main result.

Theorem 1: Consider the closed-loop system consisting of the system (1), the control law (16), the adaptive law (19) and the hysteretic quantizer (2). For any given quantized parameter $\delta \in(0,1)$, by choosing the design parameters, all signals of the closed-loop system are bounded, and the tracking error can converge to a small residual set by properly choosing the design parameters.

Proof: From Lemma 1, and note that $e b u \leq 0$, we have

$$
e d \leq-|b| \delta e u+\frac{1}{4 k_{d}} u_{\min }^{2}+k_{d} e^{2} .
$$

Then by substituting (22) into (20) and using $|e g|-e g \tanh \left(\frac{e g}{\varepsilon}\right) \leq 0.2785 \varepsilon$, we have

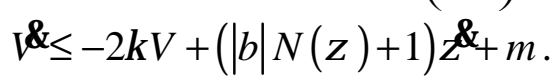

Solve the above differential equation

$$
V \leqslant \frac{m}{\kappa}+V(0)+e^{-\kappa t} \int_{0}^{t}(|b| N(\zeta)+1) \xi \varepsilon^{\kappa \tau} d \tau,
$$

where

$$
\begin{aligned}
& \kappa=\min \left\{c-k_{d}, \frac{\gamma \sigma}{2}\right\}, \\
& m=\frac{\sigma}{2} \theta^{2}+\frac{1}{4 k_{d}} u_{\min }^{2}+\eta .
\end{aligned}
$$

Using Lemma 2, we conclude that $V, \zeta$ and $\int_{0}^{t}(|b| N(\zeta)+1) \zeta d q$ are bounded. Then we have

$$
\frac{1}{2} e^{2} \leq V(t) \leq \frac{m}{2 \kappa}+\left(V(0)-\frac{m}{2 \kappa}\right) e^{-2 \kappa t}+H,
$$


where $H$ is the upper bound of $\int_{0}^{t}(|b| N(\zeta)+1) \zeta \varepsilon^{-\kappa(t-\tau)} d \tau$. Following $e$ is bounded, then by (8) and (9) we can conclude that $x_{1}, \mathrm{~K}, x_{n}$ are bounded. $v$ is bounded. Then $u$ is bounded. Furthermore, it follows that

$$
\lim _{t \rightarrow \infty} V(t) \leq \frac{m}{2 \kappa}+H
$$

It is easy to find that by choosing a sufficiently large $\kappa$, the tracking error can converge to a small residual set.

\section{Conclusions}

An adaptive quantized tracking control scheme has been proposed for a class of nonlinear systems with quantized input having unknown parameter. It is proved that by using the proposed scheme, a very coarse quantization of input signal can be obtained and all signals of the closed-loop system are bounded.

\section{References}

[1] N. Elia, S.K. Mitter, Stabilization of linear systems with limited information, IEEE Transactions on Automatic Control. 46 (2001), 1384-1400.

[2] M.Y. Fu, L.H. Xie, The sector bound approach to quantized feedback control, IEEE Transactions on Automatic Control. 50 (11) (2005), 1698-1711.

[3] T.F. Liu, Z.P. Jiang, D. Hill, A sector bound approach to feedback control of nonlinear systems with state quantization, Automatica. 48 (2012), 145-152.

[4] R.W. Brockett, D. Liberzon, Quantized feedback stabilization of linear systems, IEEE Transactions on Automatic Control. 45 (2000), 1279-1289.

[5] D. Liberzon, D. Nesic, Input-to-state stabilization of linear systems with quantized state measurements, IEEE Transactions on Automatic Control. 52 (2) (2007), 767-781.

[6] Z.P. Jiang, T.F. Liu, Quantized nonlinear control-A survey, Acta Automatica Sinica 2013. 39 (11) (2013), 1820-1830.

[7] T. Hayakawaa, H. Ishii, K. Tsumurac, Adaptive quantized control for linear uncertain discrete-time systems, Automatica. 45 (2009), 692-700.

[8] J. Zhou, C.Y. Wen, G.H. Yang, Adaptive backstepping stabilization of nonlinear uncertain systems with quantized input signal, IEEE Transactions on Automatic Control. 59 (2) (2014), 529-537. 